WU-B 00-01

PITHA 00/05

March 2000

hep-ph/0003096

\title{
Implications of light-quark admixtures on charmonium decays into meson pairs
}

\author{
Thorsten Feldmann ${ }^{1}$ and Peter Kroll ${ }^{2}$ \\ ${ }^{1}$ Institut für Theoretische Physik E, RWTH Aachen, 52056 Aachen, Germany \\ ${ }^{2}$ Fachbereich Physik, Universität Wuppertal, 42097 Wuppertal, Germany
}

\begin{abstract}
We argue that charmonium decays into meson pairs fall into two distinct classes: one that is under control of perturbative QCD and another one that is governed by a soft mechanism. We concentrate on a systematic analysis of $J / \psi\left(\Psi^{\prime}\right)$ decays into a light pseudoscalar and a light vector meson and $\eta_{c}$ decays into a pair of light vector mesons. These processes belong to the second class and are characterized by non-conserved hadronic helicity. It is assumed that, in these cases, the charmonium state decays dominantly through a lightquark Fock component by a soft mechanism which is characteristic of OZI-rule allowed strong decays. Estimating the light-quark admixture by meson mixing, we obtain a reasonable description of the branching ratios for these processes.
\end{abstract}

(submitted to Physical Review D) 


\section{Introduction}

Exclusive charmonium decays have been investigated within perturbative QCD by many

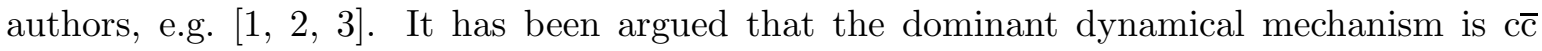
annihilation into the minimum number of gluons allowed by color conservation and charge conjugation, and subsequent creation of light quark-antiquark pairs forming the final state hadrons. This factorization of long- and short-distance physics has been shown to hold in the formal limit $m_{\mathrm{c}} \rightarrow \infty$. The dominance of annihilation through gluons is most strikingly reflected in the narrow widths of charmonium decays into hadronic channels in a mass region where strong decays typically have widths of hundreds of $\mathrm{MeV}$ [4]. Since the c and the $\bar{c}$ quarks only annihilate if their mutual distance is less than about $1 / m_{\mathrm{c}}$ (where $m_{\mathrm{c}}$ is the c-quark mass) which is smaller than the non-perturbative charmonium radius, and since the average virtuality of the gluons is of the order of $1-2 \mathrm{GeV}^{2}$ one may indeed expect perturbative QCD to be at work although corrections are presumably substantial. The charm-quark mass is too small in order to suppress power corrections decisively although it is large enough to allow perturbative QCD (pQCD) calculations. The bottomonium system, for which no exclusive hadronic decay has been observed as yet, should exhibit the pattern of perturbative predictions much cleaner.

In hard exclusive reactions higher Fock state contributions are usually suppressed by inverse powers of the hard scale, $Q$, appearing in the process $\left(Q=m_{\mathrm{c}}\right.$ for exclusive charmonium decays), as compared to the valence Fock state contribution. Hence, higher Fock state contributions are expected to be negligible in most cases. For exclusive charmonium decays, however, the valence Fock state contributions are often suppressed for one or the other reason. In such a case higher Fock state contributions or other peculiar contributions such as power corrections or small components of the hadronic wave functions may become

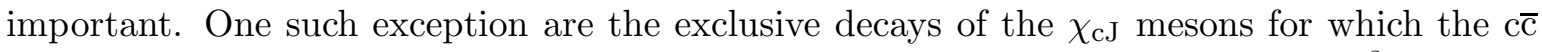
pair forms a color-singlet $\mathrm{P}$-wave state in the valence Fock state (notation: $c \bar{c}_{1}\left({ }^{3} \mathrm{P}_{\mathrm{J}}\right)$ ). As has been shown in Ref. [5] the next-higher Fock state, $c \bar{c} g$, where the quark-antiquark pair forms a $c \bar{c}_{8}\left({ }^{3} \mathrm{~S}_{1}\right)$ state, is not suppressed as compared to the valence contribution. Therefore, the neglect of the $c \bar{c} g$ contributions, which are customarily referred to as the color-octet contributions [6], is unjustified in the $\chi_{\mathrm{cJ}}$ decays. Indeed as has been discussed in Ref. [5] for the $P P$ channels (where $P$ denotes a pseudoscalar meson) and in Ref. [7] for their decays into baryon-antibaryon pairs the color-octet contributions are substantial and are definitely needed for obtaining agreement with experiment.

Decays into final states involving vector mesons $(V)$ have often peculiar properties too. On general grounds, only hadronic helicity non-conserving amplitudes contribute to some of these processes. Hence, these reactions are not under control of leading-twist pQCD. A famous example of the helicity non-conserving processes is set by the $J / \psi$ and $\Psi^{\prime}$ decays into $\rho \pi$. While $J / \psi \rightarrow \rho \pi$ has the largest branching ratio of all two-body hadronic $J / \psi$ decays, the branching ratio of $\Psi^{\prime} \rightarrow \rho \pi$ is very small [8]. The combination of these two experimental facts forms the so-called $\rho \pi$ puzzle. Many of the other $P V$ channels behave similarly. There are many attempts to understand the $J / \psi\left(\Psi^{\prime}\right) \rightarrow P V$ decays; the proposed mechanisms reach from intrinsic charm in the light mesons [9], to color-octet contributions [10], vector meson mixing [11, 12], final state interactions [13, 14] and admixtures of a glueball nearly degenerate with the $J / \psi[15,16]$. Another example of a helicity non-conserving decay is set by the $\eta_{\mathrm{c}}$ decay into proton and antiproton. In Ref. [17] it is attempted to explain this process through diquarks as quasi-elementary constituents of baryons. 
In this article we are going to attempt a systematic study of the hadronic helicity nonconserving $J / \psi\left(\Psi^{\prime}\right) \rightarrow P V$ decays. We will assume that, with a small probability, the charmonium possesses Fock components built from light quarks only. Through these Fock components the charmonium state decays by a soft mechanism. We will model this decay mechanism by $J / \psi-\omega-\phi$ mixing and subsequent $\omega$ (or $\phi$ ) decay into the $P V$ state. This, in the absence of the leading-twist perturbative QCD contribution, dominant mechanism is to be supplemented by the electromagnetic decay contribution and, depending on quantum numbers, by an anomalous, doubly Okubo-Zweig-Iizuka- (OZI-) rule violating contribution. We are not able to calculate the amplitudes of these decay processes directly but, employing flavor symmetry and mixing schemes for vector and pseudoscalar mesons, we find relations among them. With a few parameters, adjusted to experiment, we thus obtain a scheme of classification which comprises an understanding of these decay processes. The analysis of the $J / \psi\left(\Psi^{\prime}\right) \rightarrow P V$ processes along these lines is partly to be considered as an update of previous work [10, 16, 18, 19] where a general parametrization of the amplitudes for $J / \psi\left(\Psi^{\prime}\right) \rightarrow P V$ is fitted to experiment. We improve this parametrization by including new ideas on meson mixing [20] and by considering recent experimental results from the BES collaboration [21]. We also present a, partially even quantitative, physical interpretation of our parametrization which differs from those presented elsewhere [10, 16, 18]. Also new in our work is the extension of the mixing approach to the $\eta_{\mathrm{c}} \rightarrow V V$ decays.

The paper is organized as follows: Qualitative features of charmonium decays into pairs of mesons are discussed in Sec. 2. Next, in Sec. 3, we investigate the $J / \psi$ and $\Psi^{\prime}$ decays into the $P V$ channels by applying meson mixing. The mixing mechanism is also used to analyze the $\eta_{\mathrm{c}} \rightarrow V V$ decays (Sec. đ). In Sec. 5 we will comment on the mixing contributions to S-wave charmonium decays into baryons and antibaryons briefly before we present our concluding remarks in Sec. 6. Details of our treatment of vector meson mixing are given in the Appendix.

\section{Qualitative features of charmonium decays into meson pairs}

Let us consider the $P P, P V$ and $V V$ final states. A few of these channels are strictly forbidden by angular momentum and parity conservation, see Table 1. Several other channels, characterized by non-conserved naturalness, $\sigma_{\mathrm{c}} \neq \sigma_{1} \sigma_{2}$, are forbidden in pQCD to leadingtwist order; i.e. higher-twist or other dynamical mechanisms are at work here. This comes about for the following reasons: The helicity amplitudes for these processes are proportional to the totally antisymmetric $\epsilon$-tensor contracted, in all possible ways, with the available Lorentz vectors, namely the two independent momenta, $p_{1}$ and $p_{2}$, the polarization vector(s) of the light vector meson(s), $\varepsilon_{i}$, and, with the exception of the $\eta_{\mathrm{c}}$, the polarization vector or tensorfof the charmonium state. Now, in the rest frame of the decaying meson, the polarization vector of a helicity zero vector (or axial vector) meson can be expressed as a linear combination of the two final state momenta regardless whether or not the mass of the light vector meson is neglected. Hence, the number of independent Lorentz vectors is insufficient to contract the $\epsilon$-tensor with the consequence of vanishing amplitudes for processes involving longitudinally polarized vector mesons. Moreover, $\varepsilon_{1}^{*}( \pm 1)=-\varepsilon_{2}^{*}(\mp 1)$ holds in the rest frame of the decaying meson. As a consequence of these properties the only non-zero helicity amplitudes for the processes with non-conserved naturalness are those where $\lambda_{1}+\lambda_{2} \neq 0$. In other words vector

\footnotetext{
${ }^{1}$ The naturalness of a meson $i$ having spin $J_{i}$ and parity $P_{i}$ is defined as $\sigma_{i}=(-1)^{J_{i}} P_{i}$ [22].

2 Since the polarization tensor is symmetric only one Lorentz index can be contracted with the $\epsilon$-tensor.
} 


\begin{tabular}{|c||c|c|c|}
\hline & $P P$ & $P V$ & $V V$ \\
\hline \hline$\eta_{\mathrm{c}}$ & - & $(\sqrt{ })$ & $\epsilon$ \\
\hline$J / \psi$ & $(\sqrt{ })$ & $\epsilon$ & $(\sqrt{ })$ \\
\hline$\chi_{\mathrm{c} 0}$ & $\sqrt{ }$ & - & $\sqrt{ }$ \\
\hline$\chi_{\mathrm{c} 1}$ & - & $(\sqrt{ })$ & $\epsilon$ \\
\hline$\chi_{\mathrm{c} 2}$ & $\sqrt{ }$ & $(\epsilon)$ & $\sqrt{ }$ \\
\hline
\end{tabular}

Table 1: Charmonium decays into $P P, P V$ and $V V$ meson pairs. The symbols $(-, \epsilon, \sqrt{ })$ denote by angular momentum and parity conservation forbidden, in pQCD to leading-twist order forbidden and allowed channels, respectively. The brackets indicate that these channels violate either $G$-parity or isospin invariance for non-strange mesons.

mesons are transversely polarized in reactions with non-conserved naturalness. We thus conclude that in these processes hadronic helicity conservation

$$
\lambda_{1}+\lambda_{2}=0
$$

is violated. Helicity conservation is not a consequence of a particular symmetry but is a dynamical consequence of leading-twist perturbative QCD (i.e. using leading-twist wave functions and valence Fock states only): The virtual gluons from the annihilation of the cic pair create the light, (almost) massless quarks and antiquarks in opposite helicity states. To the extent that the hadronic wave functions do not embody any non-zero orbital angular momentum components, the quark helicities sum up to their parent hadron's helicity. Hence, the total helicity of the final state hadrons is zero. Consequently, processes that violate helicity conservation, are not governed by leading-twist pQCD. The remaining two-meson channels, marked by ticks in Table 1, are accessible to a perturbative treatment.

Next let us consider $G$-parity and isospin. $G$-parity or isospin-violating decays are not strictly forbidden since they can proceed through electromagnetic $c \overline{\mathrm{c}}$ annihilation and may receive contributions from the isospin-violating part of QCD. The latter contributions, being of the order of quark mass differences, seem to be small [3]. $G$-parity or isospin-violating decays of $C$-even charmonia (e.g. $\eta_{\mathrm{c}}, \chi_{\mathrm{cJ}} \rightarrow P V(J=1,2)$ for non-strange final state mesons) have not been observed experimentally as yet [8]. Proceeding on the assumption that these decays are dominantly mediated by $\mathrm{c} \overline{\mathrm{c}} \rightarrow 2 \gamma^{*} \rightarrow \mathrm{PV}$, this is understandable. They are then suppressed by a factor $\left(\alpha_{\mathrm{em}} / \alpha_{s}\right)^{4}$ as compared to the $G$-parity and isospin allowed decays of the $C$-even charmonia and their decay widths are therefore extremely small. Channels involving strange mesons (e.g. $K K^{*}$ ), should also be strongly suppressed by virtue of $U$-spin invariance. For $J / \psi$ decays the situation is different. Many $G$-parity violating (e.g. $\pi^{+} \pi^{-}$) or isospin-violating (e.g. $\omega \pi$ ) decays have been observed, the experimental branching ratios being of the order of $10^{-4}-10^{-3}$ 8]. As compared to $G$-parity and isospin allowed $J / \psi$ decays they are typically suppressed by factors of about $10^{-2}-10^{-1}$ in accordance with what is expected for an electromagnetic decay mechanism f (see Fig. 1).

That at least two distinct dynamical mechanisms are at work in exclusive charmonium decays can be realized from a comparison of $J / \psi$ and $\Psi^{\prime}$ decays. Suppose these decays

\footnotetext{
${ }^{3}$ Somewhat exceptional is the $\chi_{\mathrm{c} 1} \rightarrow V V$ case where one out of the three spin-1 mesons may be longitudinally polarized.

${ }^{4}$ A possible correction due to $c \overline{\mathrm{c}}$ annihilation mediated by $\gamma^{*} g^{*} g^{*}$ is ignored by us here.
} 


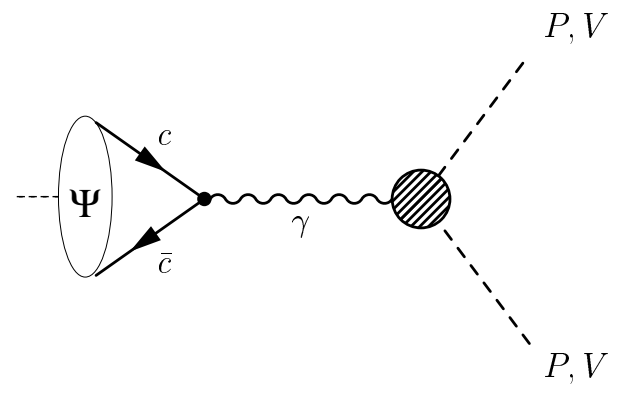

Figure 1: Electromagnetic $J / \psi$ and $\Psi^{\prime}$ decays into meson pairs. The shaded circle indicates the time-like electromagnetic (transition) form factor of light vector and/or pseudoscalar mesons.

are under control of a mechanism that respects QCD factorization and where the charm and anticharm quarks annihilate into gluons and/or photons at small mutual distance. The charmonium wave function is then probed at small spatial separations and, therefore, is well represented by the corresponding decay constant, $f_{J / \psi}$ or $f_{\Psi^{\prime}}$, which is measured in electronic decays of that charmonium state. If this short distance mechanism is responsible for the $J / \psi$ and $\Psi^{\prime}$ decay into a particular two hadron channel, the ratio

$$
\kappa_{12}=\frac{\mathcal{B}\left(\Psi^{\prime} \rightarrow h_{1} h_{2}\right)}{\mathcal{B}\left(J / \psi \rightarrow h_{1} h_{2}\right)} \frac{\mathcal{B}\left(J / \psi \rightarrow e^{+} e^{-}\right)}{\mathcal{B}\left(\Psi^{\prime} \rightarrow e^{+} e^{-}\right)}\left(\frac{\varrho_{12}(J / \psi)}{\varrho_{12}\left(\Psi^{\prime}\right)}\right)^{n}
$$

should be close to unity ( $n=3$ for $P V$ channels and $n=1$ otherwise). Since $\mathcal{B}\left(\Psi^{\prime} \rightarrow\right.$ $\left.e^{+} e^{-}\right) / \mathcal{B}\left(J / \psi \rightarrow e^{+} e^{-}\right)$amounts to about 14\%, the relation (2) is occasionally termed the $14 \%$ rule. The phase space factor in Eq. (2) is defined by

$$
\varrho_{12}(j)=\sqrt{1-2\left(m_{1}^{2}+m_{2}^{2}\right) / M_{j}^{2}+\left(m_{1}^{2}-m_{2}^{2}\right)^{2} / M_{j}^{4}}
$$

where $M_{j}$ is the mass of the charmonium state. It is of numerical relevance only for particles with masses above $1 \mathrm{GeV}$. Experimental results for $\kappa$ are listed in Table 2; the data are taken from Refs. [8, 21, 24, 25, 26, 27]. For comparison we also include experimental results for the baryon-antibaryon, vector-tensor and axial-vector-pseudoscalar channels in the table. We observe that, within often large errors or only within bounds, $\kappa$ is indeed compatible with unity for the baryon-antibaryon channels, for $b_{1} \pi, K^{* 0} K^{* 0}$ and for some of the $P V$ channels, notably for the $G$-parity and isospin-violating channels $\pi \omega, \eta \rho, \eta^{\prime} \rho$. For most of the other $P V$ channels and, perhaps, to a lesser extent also for the vector-tensor channels, $\kappa$ is well below unity. We stress that the decays into a vector and a tensor meson are not forbidden by hadronic helicity conservation, but leading-twist pQCD feeds the helicity amplitude $\mathcal{M}_{00 \lambda_{c}}$ only. There are many other, in general non-zero amplitudes that do not respect hadronic helicity conservation. Note that the branching ratios of $J / \psi$ decays into $V T$ are large, comparable with those for the $P V$ channels while those for the corresponding $\Psi^{\prime}$

\footnotetext{
${ }^{5}$ In a consistent perturbative calculation to lowest order in the c-quark velocity expansion [5, 6, 23] one has to evaluate the hard scattering amplitude from $2 m_{\mathrm{c}}$ instead from the bound state mass; the mass difference is an effect of order $v^{2}$, i.e. of the same order as the contributions from the next higher Fock states. An additional factor of $\left(M_{\Psi^{\prime}} / M_{J / \psi}\right)^{2 n}$ in Eq. (2) ( $n=4$ for baryon-antibaryon channels for instance), to be found in the literature occasionally, is inconsistent in this respect.
} 


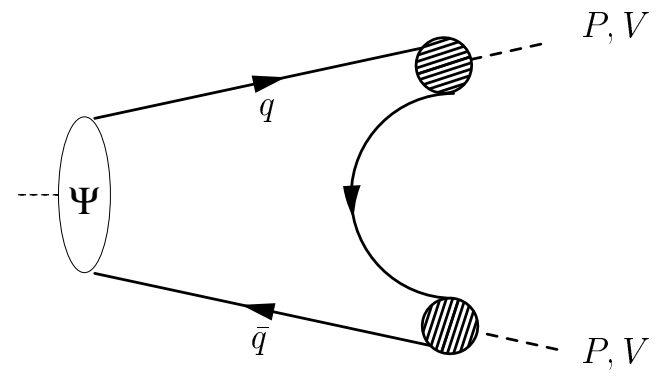

Figure 2: Schematic picture of the mixing mechanism for charmonium decays into meson pairs.

decays are small. For the $P P$ channels the situation is unclear; better data are demanded. We do not include the data for multi-particle channels in the table but we note that, with the exception of the final states consisting of three pseudoscalars, $\kappa$ is compatible with unity for all these channels. This fact is presumably a consequence of an underlying perturbatively generated three gluon jet structure which fragments into multi-particle final states. This interpretation is supported by estimates of the total width for $J / \psi$ decays into light hadrons through the decay into three real gluons [28]. In the case of the $\Upsilon$ decays the three jet structure is experimentally established [29].

The small value of $\kappa$ for those $P V$ channels that are dominated by strong interactions, arises from large $J / \psi$ branching ratios as an inspection of Table 2 reveals. The $\Psi^{\prime}$ decays into $P V$, on the other hand, seem to behave, at least in tendency, according to expectations based on pQCD; their branching ratios are smaller than those for the leading-twist allowed channels. Obviously, another dynamical mechanism is called for which is active for the $J / \psi$ decays but suppressed for the $\Psi^{\prime}$ ones. For this mechanism we assume that the charmonium state possesses Fock components built from light quarks only. It can then decay through these Fock components by a soft mechanism that is characteristic of OZI-rule allowed strong

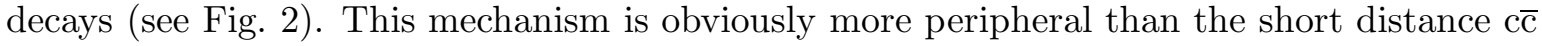
annihilation; i.e. it probes the charmonium wave function at all quark-antiquark separations and feels therefore the difference between a $1 \mathrm{~S}$ and a $2 \mathrm{~S}$ radial wave function. The node in the latter is supposed to lead to a strong suppression of this mechanism in the $\Psi^{\prime}$ decays. We model this mechanism by mixing of vector mesons, $J / \psi-\omega-\phi$. For the mixing angle, $\tilde{\theta}_{\mathrm{c}}$, being of order $1 / m_{\mathrm{c}}^{2}$, we take the value $-0.1^{\circ}$ which corresponds to a light-quark admixture to the charmonium state of order of $10^{-3}$, see the Appendix. The light vector mesons, $\omega$ and $\phi$, couple to the final state mesons through vertex functions, e.g. $g_{\omega V} P$. In terms of vector meson mixing the suppression of the $\Psi^{\prime} \rightarrow P V$ decays follows from substantially weaker couplings (arguments in favor of that assumption are given in Refs. [12, 31]) of the radially excited $\omega^{\prime}$ and $\phi^{\prime}$ mesons (assumed to mix with the $\Psi^{\prime}$ ) to the ground state pseudoscalar and vector mesons and, perhaps, from a smaller mixing angle. The mixing mechanism may also be at work in the vector-tensor channels (with vertex functions $g_{V V T}$ ) and may generate the small value of $\kappa$ there, see Table 2 .

Conversely one may also consider the ce Fock components of $\omega$ and $\phi$ mesons in the final

\footnotetext{
${ }^{6}$ This assumption bears a resemblance to the node effect discussed in Ref. 30.
} 
state; their probablilities are the same as the ones for light quark components in the $J / \psi$ meson, see, Eq. (31) in the appendix. The initial c $\bar{c}$ pair from the $J / \psi$ may then feed that Fock component of the $\omega$ or $\phi$. However, the second final state meson is to be generated by an OZI-rule violating mechanism now, and is to be considered as a higher-order correction to the leading mixing mechanism we propose. For channels like $\omega \eta^{\prime}$ one may also think of an excitation of the $c \bar{c}$ components of both the light mesons. Obviously, the probability of finding a $c \overline{\mathrm{c}}$ pair in a light meson enters quadratically now, and this decay mechanism is suppressed by an additional power of $1 / m_{c}^{2}$. Hence, we ignore both the above possibilities.

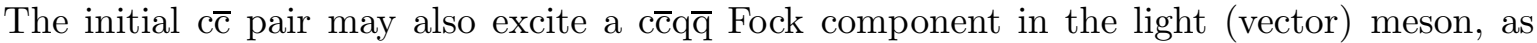
suggested by Brodsky and Karliner [9]. In that picture the q $\bar{q}$ pair from this Fock component forms the other final state meson. Of course, one expects the probability of this higher Fock state to be very small. In any case the approach of Brodsky and Karliner should be viewed as complementary to ours, since the mechanism that they propose cannot be represented by meson-mixing supplied with OZI-rule allowed decay vertices.

We note that in Refs. [10, 16, 18] the strong interaction mechanism for the hadronic helicity non-conserving decays although parametrized in a similar way as we do, is interpreted differently. Thus, Chen and Braaten [10] argue that this contribution is generated by an additional Fock state gluon (the c c pair is therefore in a color-octet state) which carries a unit of helicity from the $J / \psi$ to one of the light mesons without participating in the hard process. Chen and Braaten provide arguments that the suppression of the $\Psi^{\prime}$ decays into $P V$ mesons as well as into the vector-tensor channels is due to the energy gap between the mass of the $\Psi^{\prime}$ and the $D \bar{D}$ threshold. In Refs. [16, 18], on the other hand, mixing of the $J / \psi$ with a fairly narrow $J^{P C}=1^{--}$glueball, nearly degenerate with the $J / \psi$, is assumed. The $J / \psi$ can then decay through the glueball while this mechanism is not available for the $\Psi^{\prime}$. Searches for such a glueball, performed by the BES collaboration [32], however turned out negative although not fully conclusive to claim the demise of the glueball interpretation. For the final state interaction mechanism advocated in Refs. [13, 14] the suppression of the $\Psi^{\prime} \rightarrow P V$ decays is due to a fortuitous cancellation of various contributions and, probably, does not hold for the vector-tensor channels.

The electromagnetic decay mechanism, proceeding through the subprocess $c \overline{\mathrm{c}} \rightarrow \gamma^{*} \rightarrow$ $\mathrm{h}_{1} \mathrm{~h}_{2}$ (see Fig. 1), also probes the charmonium wave function at small spatial separation and, hence, would lead to $\kappa \simeq 1$ if it dominates. This is what we observe, or at least what is indicated, by the isospin-violating $\pi \omega, \eta \rho$ and $\eta^{\prime} \rho$ channels. For the other $J / \psi\left(\Psi^{\prime}\right) \rightarrow P V$ decays a superposition of the mixing and the electromagnetic mechanism occurs. For the flavor-singlet channels one has to consider an anomalous (formally doubly OZI-rule violating) contribution [18, 19] too, see Fig. 3. The mixing mechanism also applies to the $\eta_{\mathrm{c}} \rightarrow V V$ decays with vector meson mixing replaced by that for pseudoscalar mesons [20].

The $\chi_{\mathrm{cJ}}$ may also decay through light-quark Fock components. However, in terms of meson mixing, the model would require the coupling of appropriate $0^{++}, 1^{++}, 2^{++}$mesons to pairs of ground state mesons. These couplings are expected to be small and, therefore, the $\chi_{\mathrm{c} J} \rightarrow P P, V V(J=0,2)$ decays seem to be dominated by perturbative contributions while the hadronic helicity non-conserving decays $\chi_{\mathrm{c} 1} \rightarrow V V$ should be strongly suppressed. Indeed, the latter decays have not been observed experimentally as yet [8]. As we already mentioned in the introduction the perturbative analysis [5, 23] of the $\chi_{\mathrm{cJ}}$ decays into the $P P$ channels provides results in fair agreement with experiment [8, 33]. In this analysis, it is however important to include the color-octet contributions, i.e. the $c \bar{c} g$ Fock state of the $\chi_{\mathrm{cJ}}$. The contribution of the valence Fock state alone, evaluated from the asymptotic form 


\begin{tabular}{|c|c|c|c|}
\hline channel & $10^{4} \mathcal{B}(J / \psi)$ & $10^{4} \mathcal{B}\left(\Psi^{\prime}\right)$ & $\kappa$ \\
\hline$p \bar{p}$ & $21.4 \pm 1.0$ & $2.62 \pm 0.57$ & $0.89 \pm 0.20$ \\
\hline & & $1.9 \pm 0.5 \quad \mathrm{PDG}$ & $0.64 \pm 0.18$ \\
\hline$\Sigma^{0} \bar{\Sigma}^{0}$ & $12.7 \pm 1.7$ & $1.20 \pm 0.50$ & $0.63 \pm 0.33$ \\
\hline$\Lambda \bar{\Lambda}$ & $13.5 \pm 1.4$ & $1.89 \pm 0.29$ & $0.99 \pm 0.20$ \\
\hline$\Xi^{-\bar{\Xi}^{+}}$ & $9.0 \pm 2.0$ & $1.00 \pm 0.32$ & $0.65 \pm 0.35$ \\
\hline$\Delta^{++} \bar{\Delta}^{--}$ & $11.0 \pm 2.9$ & $1.34 \pm 0.35$ & $0.78 \pm 0.28$ \\
\hline$\pi^{+} \pi^{-}$ & $1.47 \pm 0.23$ & $0.8 \pm 0.5 \mathrm{PDG}$ & $4.25 \pm 2.80$ \\
\hline$K^{+} K^{-}$ & $2.37 \pm 0.31$ & $1.0 \pm 0.7 \mathrm{PDG}$ & $3.25 \pm 2.30$ \\
\hline$\rho \pi$ & $128 \pm 10$ & $<0.28$ & $<0.016$ \\
\hline & & $<0.83 \mathrm{PDG}$ & $<0.048$ \\
\hline$K^{+} \bar{K}^{*-}+c . c$. & $50.0 \pm 4.0$ & $<0.3$ & $<0.042$ \\
\hline & & $<0.54 \mathrm{PDG}$ & $<0.075$ \\
\hline$K^{0} \bar{K}^{* 0}+c . c$. & $42.0 \pm 4.0$ & $0.81 \pm 0.29$ & $0.13 \pm 0.06$ \\
\hline$\omega \eta$ & $15.8 \pm 1.6$ & $<0.33$ & $<0.15$ \\
\hline$\omega \eta^{\prime}$ & $1.67 \pm 0.25$ & $0.76 \pm 0.48$ & $2.94 \pm 1.90$ \\
\hline$\phi \eta$ & $6.5 \pm 0.7$ & $0.35 \pm 0.20$ & $0.36 \pm 0.21$ \\
\hline$\phi \eta^{\prime}$ & $3.3 \pm 0.4$ & $<0.75$ & $<1.35$ \\
\hline$\rho \eta$ & $1.93 \pm 0.23$ & $0.21 \pm 0.12$ & $0.77 \pm 0.45$ \\
\hline$\rho \eta^{\prime}$ & $1.05 \pm 0.18$ & $<0.3$ & $<1.84$ \\
\hline$\omega \pi$ & $4.2 \pm 0.6$ & $0.38 \pm 0.20$ & $0.67 \pm 0.37$ \\
\hline$K^{* 0} K^{* 0}$ & $<5.0$ & $0.45 \pm 0.26$ & $>0.66$ \\
\hline$\omega f_{2}$ & $43.0 \pm 6.0$ & $<1.7$ & $<0.28$ \\
\hline$\rho a_{2}$ & $109 \pm 22$ & $<2.3$ & $<0.15$ \\
\hline$K^{* 0} \bar{K}_{2}^{* 0}+$ c.c. & $67.0 \pm 26$ & $<1.2$ & $<0.12$ \\
\hline$\phi f_{2}^{\prime}$ & $8.0 \pm 4.0$ & $<0.45$ & $<0.45$ \\
\hline$b_{1} \pi+c . c$. & $30.0 \pm 5.0$ & $5.3 \pm 1.2$ & $1.28 \pm 0.38$ \\
\hline$K_{1}(1270) K^{-}+c . c$. & $<29 \mathrm{BES}$ & $10.0 \pm 5.0$ & $>2.48$ \\
\hline$K_{1}(1400) K^{-}+$c.c. & $38.0 \pm 9.5$ & $<2.9$ & $<0.54$ \\
\hline
\end{tabular}

Table 2: Exclusive $J / \psi$ and $\psi^{\prime}$ branching ratios and their ratios scaled by the corresponding electronic branching ratios and phase space corrected according to Eq. (2). Data are taken from [8] in the $J / \psi$ cases and from the BES collaboration [21, 24, 25, 26] if not stated otherwise. Some of the BES data are preliminary; the BES data on $\Psi^{\prime} \rightarrow \rho \eta^{(\prime)}, \phi \eta^{(\prime)}$ are quoted in Ref. [27]. 


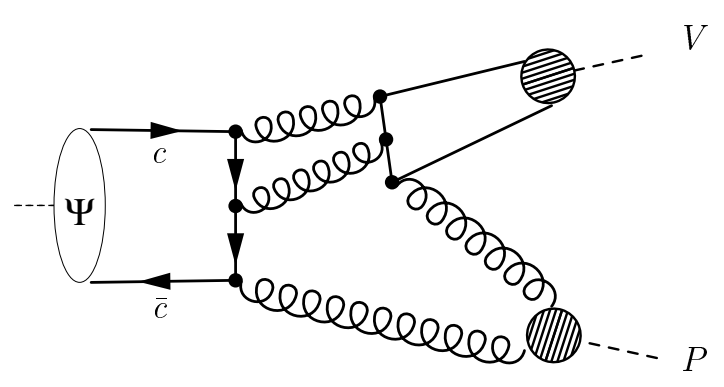

Figure 3: Schematic picture of the anomalous (doubly OZI-rule violating) decay mechanism for $J / \psi$ and $\Psi^{\prime}$ decays into $P V$ meson pairs.

of the pseudoscalar meson's light-cone wave function (as determined from the analysis of the $P \gamma$ transition form factors [34, 35, 36]), is substantially smaller than experiment. Aspects of the $\eta-\eta^{\prime}$ mixing in these decays have been discussed in Ref. [37]. The extension of the perturbative analysis to the $V V$ channels, for which first data exist [33], is straightforward but the required wave functions of the vector mesons are not known to a sufficient degree of accuracy at present.

\section{Mixing mechanism and the decays $J / \psi\left(\Psi^{\prime}\right) \rightarrow P V$}

Let us now turn to the $J / \psi$ and $\Psi^{\prime}$ decays into a pseudoscalar and a vector meson. According to the discussion presented in Sec. 2 we take into account the three mechanisms shown in Figs. 1, 2 and 2. Each of these contributions will be parametrized by a reduced amplitude, common to all $P V$ channels, multiplied by flavor factors, meson-mixing factors and flavorsymmetry corrections. Because of the lack of a compelling theory we have to treat the reduced amplitudes as free parameters to be adjusted to experiment. Despite this fact our ansatz which is structurally similar, although different in detail, to those discussed in Refs. [10, 16, 18, 19] provides a systematic description of the $J / \psi$ and $\Psi^{\prime}$ decays into $P V$ mesons as we will see shortly.

To begin with we discuss the electromagnetic decay mechanism shown in Fig. 1. Decomposing the helicity amplitudes for a decay of an $n^{3} S_{1}$ charmonium state into $P V$ covariantly as

$$
\mathcal{M}_{0 \lambda_{V}, \lambda}\left(n^{3} \mathrm{~S}_{1} \rightarrow V P\right)=\frac{A_{P V}\left(n^{3} \mathrm{~S}_{1}\right)}{M^{2}\left(n^{3} \mathrm{~S}_{1}\right)} \epsilon\left(p_{1}, p_{2}, \varepsilon_{V}^{*}\left(\lambda_{V}\right), \varepsilon(\lambda)\right)
$$

we find for the electromagnetic contribution to the invariant amplitude

$$
A_{P V}^{\mathrm{em}}\left(n^{3} \mathrm{~S}_{1}\right)=4 \pi \alpha_{\mathrm{em}} e_{\mathrm{c}} e^{i \theta_{e}} f\left(n^{3} \mathrm{~S}_{1}\right) M\left(n^{3} \mathrm{~S}_{1}\right) F_{P V}(s) .
$$

$f\left(n^{3} \mathrm{~S}_{1}\right)$ is the decay constant of the $n^{3} \mathrm{~S}_{1}$ charmonium state. Its numerical value is taken from Ref. [38] $\left(f(J / \psi)=405 \mathrm{MeV}\right.$ and $\left.f\left(\Psi^{\prime}\right)=282 \mathrm{MeV}\right) . F_{P V}$ is the time-like $P \rightarrow V$ transition form factor for transversely polarized vector mesons at $s=M^{2}\left(n^{3} \mathrm{~S}_{1}\right)$. We write this form factor as

$$
F_{P V}(s)=c_{P V}^{\mathrm{em}} F_{\rho^{0} \pi^{0}}(s)
$$




\begin{tabular}{|c||c|c|c|}
\hline$P V$ & $c_{P V}^{\mathrm{em}}$ & $c_{P V}^{\mathrm{mix}}$ & $c_{P V}^{\mathrm{anom}}$ \\
\hline \hline$\omega \pi$ & 3 & - & - \\
$\rho \eta$ & $3 \cos \phi_{P}$ & - & - \\
$\rho \eta^{\prime}$ & $3 \sin \phi_{P}$ & - & - \\
$\phi \pi$ & $3 \phi_{V}$ & - & - \\
\hline$\rho^{0, \pm} \pi^{0, \mp}$ & 1 & 1 & $-\sqrt{2} \tan \theta_{8}$ \\
$\omega \eta$ & $\cos \phi_{P}$ & $\cos \phi_{P}$ & $\sqrt{2}$ \\
$\omega \eta^{\prime}$ & $\sin \phi_{P}$ & $\sin \phi_{P}$ & - \\
\hline$K^{*+} K^{-}$ & $y^{\mathrm{em}}$ & $\left(\tilde{y}-\sqrt{2} \cot \tilde{\theta}_{y}\right) / 2$ & - \\
$K^{* 0} \bar{K}^{0}$ & $-2 y^{\mathrm{em}}$ & $\left(\tilde{y}-\sqrt{2} \cot \tilde{\theta}_{y}\right) / 2$ & -2 \\
$\phi \eta$ & $2 \sin \phi_{P}\left(y^{\mathrm{em}}\right)^{2}$ & $\sqrt{2} \sin \phi_{P} \cot \tilde{\theta}_{y} \tilde{y}$ & $-\tilde{y} \tan \theta_{8}$ \\
$\phi \eta^{\prime}$ & $-2 \cos \phi_{P}\left(y^{\mathrm{em}}\right)^{2}$ & $-\sqrt{2} \cos \phi_{P} \cot \tilde{\theta}_{y} \tilde{y}$ & $\tilde{y}$ \\
\hline
\end{tabular}

Table 3: The flavor factors appearing in the amplitudes for $n^{3} S_{1} \rightarrow P V$ decays. For definitions see Eqs. (6, 10, 12). The parameters for $\eta-\eta^{\prime}$ mixing, $\phi_{P}$ and $\theta_{8}$, are defined in Ref. [20], those describing vector meson mixing, $\phi_{V}, \tilde{\theta}_{y}, \tilde{y}$, in the Appendix.

and treat $F_{\rho^{0} \pi^{0}}(s)$ as a free parameter $\left(c_{\rho^{0} \pi^{0}}^{\mathrm{em}}=1\right.$ by definition $) . c_{P V}^{\mathrm{em}}$ depends on the electric charges of the $P V$ system in units of the elementary charge $e_{0}$. We also absorb $\eta-\eta^{\prime}$ and $\omega-\phi$ mixing factors as well as a flavor symmetry breaking correction factor, $y^{\mathrm{em}}$, into $c_{P V}^{\mathrm{em}}$. The parameter $y^{\mathrm{em}}$ takes into account the difference between the occurrence of strange and non-strange quarks in the form factors; its value will be estimated below. The mixing of the pseudoscalar mesons is described in the quark-flavor scheme advocated in Ref. [20]. Vector meson mixing is treated analogously, the details are presented in the Appendix. Since the vector meson mixing angle, $\phi_{V}$, is so small (see Eq. (30)) we put $\cos \phi_{V} \simeq 1$ and $\sin \phi_{V} \simeq \phi_{V}$. The flavor factors $c_{P V}^{\mathrm{em}}$ are compiled in Table 3. In Eq. (5) we also allow for a phase, $\theta_{e}$, relative to the strong interaction contributions discussed below. The transition form factors are therefore to be understood as absolute values. The invariant function $A_{P V}$ in Eq. (雨) is normalized in such a way that the decay width reads

$$
\Gamma\left(n^{3} \mathrm{~S}_{1} \rightarrow P V\right)=\frac{1}{96 \pi} \frac{\varrho_{P V}\left(n^{3} \mathrm{~S}_{1}\right)^{3}}{M\left(n^{3} \mathrm{~S}_{1}\right)}\left|A_{P V}\right|^{2} .
$$

The four isospin-violating channels now allow a simple extraction of the $\rho^{0} \pi^{0}$ form factor. From a fit to the experimental branching ratios of the isospin-violating decay channels we find

$$
\begin{aligned}
F_{\rho^{0} \pi^{0}}\left(M_{J / \psi}^{2}\right) & =27 \cdot 10^{-3} \mathrm{GeV}^{-1} \\
F_{\rho^{0} \pi^{0}}\left(M_{\Psi^{\prime}}^{2}\right) & =19 \cdot 10^{-3} \mathrm{GeV}^{-1} .
\end{aligned}
$$

The results of the fit are listed in Table 14 . Note that the values (8) of the form factors are subject to errors which we estimate to $7.5 \%$ and $21 \%$ for the $J / \psi$ and $\Psi^{\prime}$ cases, respectively. The calculation of the time-like form factor, in particular in the vicinity of resonances, is a delicate issue and, in so far, reliable theoretical information on this form factor is lacking. Since the $\rho$ meson is transversely polarized, the form factor is under control of higher-twist contributions and should therefore fall off as $1 / s^{2}$ asymptotically. Comparison of the two 


\begin{tabular}{|c||cc||cc|}
\hline$P V$ & $10^{4} \cdot \mathcal{B}_{\text {th }}(J / \psi)$ & $10^{4} \cdot \mathcal{B}_{\exp }(J / \psi)$ & $10^{4} \cdot \mathcal{B}_{\text {th }}\left(\Psi^{\prime}\right)$ & $10^{4} \cdot \mathcal{B}_{\exp }\left(\Psi^{\prime}\right)$ \\
\hline \hline$\omega \pi$ & 3.78 & $4.20 \pm 0.60$ & 0.38 & $0.38 \pm 0.20$ \\
$\rho \eta$ & 2.04 & $1.93 \pm 0.23$ & 0.21 & $0.21 \pm 0.12$ \\
$\rho \eta^{\prime}$ & 1.06 & $1.05 \pm 0.18$ & 0.12 & $<0.3$ \\
$\phi \pi$ & 0.013 & $<0.068$ & 0.001 & - \\
\hline$\rho \pi$ & 127 & $128 \pm 10$ & 0.13 & $<0.28$ \\
$\omega \eta$ & 14.7 & $15.8 \pm 1.6$ & 0.12 & $<0.33$ \\
$\omega \eta^{\prime}$ & 1.86 & $1.67 \pm 0.25$ & 0.63 & $0.76 \pm 0.48$ \\
\hline$K^{*+} K^{-}+$c.c. & 48 & $50 \pm 4$ & 0.13 & $<0.30$ \\
$K^{* 0} \bar{K}^{0}+$ c.c. & 42 & $42 \pm 4$ & 0.51 & $0.81 \pm 0.29$ \\
$\phi \eta$ & 7.5 & $6.5 \pm 0.7$ & 0.16 & $0.35 \pm 0.20$ \\
$\phi \eta^{\prime}$ & 2.6 & $3.3 \pm 0.4$ & 0.46 & $<0.75$ \\
\hline
\end{tabular}

Table 4: Comparison of predictions and experiment for the branching ratios (multiplied by $10^{4}$ ) for $J / \psi$ and $\Psi^{\prime}$ decays into a pseudoscalar and a vector meson. The results for the first two sets of decay channels are obtained from fits (see text). The third set of decay channels involving strange mesons are predictions based on the parameters determined in the fits to the first two sets of data. The $J / \psi$ data are the PDG averages [8], the $\Psi^{\prime}$ ones are preliminary BES data [21, 24, 27]. The values of the mixing parameters for vector mesons are quoted in the Appendix, those for pseudoscalar mesons are $\phi_{P}=39.3^{\circ}$ and $\theta_{8}=-21.2^{\circ}$ [20].

values in Eq. (8) rather reveals an approximate $1 / s$ behavior in the considered region of $s$. A recent QCD sum rule analysis [39] of the space-like $\rho \pi$ form factor yields values that are somewhat smaller than those quoted in Eq. (8). The enhancement of time-like form factors relative to space-like ones is known from the pion and nucleon cases; the disparities typically amount to about a factor of 3 in the charmonium region (see e.g. [17, 40]). In any case, measurements of the $P V$ transition form factors in the charmonium region would be welcome. Such data would permit a critical examination of the interpretation of the electromagnetic contribution (5) (cf. also the discussion in Ref. [16]). In particular it might be checked whether or not the neglect of contributions from the isospin-violating part of QCD and from $c \overline{\mathrm{c}}$ annihilation mediated by $\gamma^{*} g^{*} g^{*}$ is indeed justified.

The $\phi \pi$ channel offers direct possibility of testing $\omega-\phi$ mixing. As inspection of Table 1 reveals the predicted $J / \psi \rightarrow \phi \pi$ branching ratio obtained from our value of the mixing angle (30), is well in agreement with the experimental bound. A measurement of the branching ratio for the $\phi \pi$ channel would allow a direct determination of $\phi_{V}$ through the ratio

$$
\frac{\mathcal{B}\left(n^{3} S_{1} \rightarrow \phi \pi\right)}{\mathcal{B}\left(n^{3} S_{1} \rightarrow \omega \pi\right)}=\phi_{V}^{2}\left(\frac{\varrho_{\omega \pi}\left(n^{3} S_{1}\right)}{\varrho_{\phi \pi}\left(n^{3} S_{1}\right)}\right)^{3},
$$

a relation that was proposed by Haber and Perrier [41] long time ago.

The invariant amplitude for the strong decay mechanism (see Fig. 2) is parametrized as

$$
A_{P V}^{\operatorname{mix}}\left(n^{3} S_{1}\right)=c_{P V}^{\operatorname{mix}} g^{\operatorname{mix}}\left(n^{3} S_{1}\right)
$$

where $c_{P V}^{\operatorname{mix}}$ depends on flavor symmetry breaking and meson mixing factors. As for the electromagnetic contribution we put $c_{\rho_{0} \pi_{0}}^{\mathrm{mix}}=1$. By virtue of the node effect (see the discussion 
in Sec. 2) this mechanism does not contribute to the $\Psi^{\prime}$ decays, i.e. we assume $g^{\operatorname{mix}}(J / \psi) \gg$ $g^{\operatorname{mix}}\left(\Psi^{\prime}\right) \simeq 0$ for the reduced amplitude.

The anomalous contribution, depicted in Fig. 3, can only contribute to the flavor singlet meson channels. It is assumed to proceed via a short-distance annihilation of the initial c $\bar{c}$ pair and a subsequent creation of the $\eta$ and $\eta^{\prime}$ states controlled by the matrix element of the topological charge density $\left\langle 0\left|\frac{\alpha_{s}}{4 \pi} G \tilde{G}\right| \eta^{(\prime)}\right\rangle$ which incorporates the $\mathrm{U}(1)_{\mathrm{A}}$ anomaly. As shown in Refs. [20, 37] the ratio of both the gluon matrix elements is given by

$$
\frac{\left\langle 0\left|\frac{\alpha_{s}}{4 \pi} G \tilde{G}\right| \eta\right\rangle}{\left\langle 0\left|\frac{\alpha_{s}}{4 \pi} G \tilde{G}\right| \eta^{\prime}\right\rangle}=-\tan \theta_{8},
$$

where the angle $\theta_{8}$ is related to the $\eta-\eta^{\prime}$ mixing angle $\phi_{P}$ via $\theta_{8}=\phi_{P}-\arctan [\sqrt{2} / y]$, where $y$ being the ratio of basic decay constants, $f_{\mathrm{q}}$ and $f_{\mathrm{s}}$ in the quark-flavor scheme [20]. The numerical values for the mixing parameters, determined in Ref. [20], read $\phi_{P}=39.3^{\circ}$, $\theta_{8}=-21.2^{\circ}$ and $y=0.81$. In view of these considerations we parametrize the anomalous contribution as

$$
A_{P V}^{\text {anom }}\left(n^{3} S_{1}\right)=f\left(n^{3} S_{1}\right) c_{P V}^{\text {anom }} g^{\text {anom }}\left(n^{3} S_{1}\right),
$$

and define $c_{\omega \eta^{\prime}}^{\text {anom }}=\sqrt{2}$. The flavor factors $c_{P V}^{\text {anom }}$ for the other channels can again be found in Table 3. Because of the short distance $c \bar{c}$ annihilation mechanism $g^{\text {anom }}$, the reduced amplitude of the anomalous contribution, should only mildly depend on the charmonium mass; we therefore assume $g^{\text {anom }}(J / \psi)=g^{\text {anom }}\left(\Psi^{\prime}\right)$ for simplicity.

A fit to the $J / \psi$ and $\Psi^{\prime}$ decay widths into $\rho \pi, \omega \eta$ and $\omega \eta^{\prime}$ provides the results shown in Table 4 for the following values of the parameters

$$
\begin{aligned}
& g^{\operatorname{mix}}(J / \psi)=0.020 \mathrm{GeV} \\
& g^{\text {anom }}=-0.014 \\
& \theta_{e}=78^{\circ}
\end{aligned}
$$

The value of the relative angle between the electromagnetic and the strong contributions is very similar to that found by Bramon et al. [19]. Relative phases with values close to $90^{\circ}$ between different decay amplitudes have also been observed in other approaches to $J / \psi\left(\psi^{\prime}\right)$ decays [14, 42, 43]. Concerning the relative size of contributions from the three individual decay mechanisms (electromagnetic, mixing, anomalous), the result strongly depends on the considered decay channel. For instance, in the case of $J / \psi \rightarrow \rho \pi$ the ratio of $\left|A^{\mathrm{em}}\right| /\left|A^{\mathrm{mix}}\right|$ is only 0.1 whereas for $J / \psi \rightarrow K^{* 0} \bar{K}^{0}$ the same ratio is about 0.3 . The anomalous contribution tends to interfere with the mixing contribution destructively and is in some cases very important. This is most drastically seen in the channel $J / \psi \rightarrow \phi \eta^{\prime}$ where the ratio $\left|A^{\mathrm{em}}\right| /\left|A^{\mathrm{mix}}+A^{\text {anom }}\right|$ is about 1.1 .

The decays into $K K^{*}$ and $\phi \eta\left(^{\prime}\right)$ involve strange quarks which necessitates the consideration of flavor symmetry breaking. In the case of the electromagnetic contribution one has to pay attention to the fact that the $P V$ transition form factors are controlled by higher-twist contributions since the vector mesons are transversely polarized. Hence, the form factors are proportional to a mass scale which, as shown by Efremov and Teryaev [44], is of the order of the hadron masses and not, as one may naively expect, set by current-quark masses. Modelling this mass scale by appropriate effective constituent-quark masses $(\hat{m})$, we estimate $y^{\mathrm{em}} \simeq \hat{m}_{\mathrm{s}} / \hat{m}_{\mathrm{q}} \simeq 1.3$. For $\phi \eta$ and $\phi \eta^{\prime}$ channels the factor should appear quadratically. For 
the mixing contribution strange quarks come either from $J / \psi-\phi$ mixing, and are therefore $\propto \cot \tilde{\theta}_{y}$, or by a soft creation of a s $\bar{s}$ pair out of the vacuum. This creation process is suppressed by a factor that is less than unity, relative to the creation of a non-strange quarkantiquark pair. In accordance with the flavor symmetry breaking in vector meson mixing, see the Appendix, we identify this factor with $\tilde{y}$ and use the value 0.8 for it. Note that in our mixing scheme $\sqrt{2} \cot \tilde{\theta}_{y}=-\tilde{y}$, see Eq. (25). Therefore, the pattern of $\mathrm{SU}(3)_{\mathrm{F}}$ breaking is similar for the mixing and the electromagnetic contributions. With these values for the flavor symmetry factors and the other already determined parameters at hand we are in the position to predict the branching ratios for the decay channels involving strange quarks. The results are also shown in Table 1 . The quality of the predictions for the entire set of $J / \psi$ and $\Psi^{\prime}$ decays into a $P V$ pair is good. For the $J / \psi$ decays the quality is similar to that obtained in Ref. [19] although we have a smaller number of adjustable parameters.

In our approach the $\Psi^{\prime}$ decays into the $P V$ pair electromagnetically with the exception of the flavor-singlet channels where the anomalous mechanism contributes as well. In so far our analysis differs from that advocated by Tuan [16] who extended the analysis in Ref. [19] to the case of the $\Psi^{\prime}$. Tuan assumes the electromagnetic and the strong contributions to cancel approximately in the $\Psi^{\prime} \rightarrow \rho \pi$ channel. This assumption leads to substantially smaller branching ratios for the $\Psi^{\prime} \rightarrow \rho \pi$ and $\Psi^{\prime} \rightarrow K^{*+} K^{-}$channels than we obtain. Since for both channels the BES collaboration only provides bounds the issue which of the contending models is correct, cannot be settled as yet.

Let us now dwell upon the compatibility of the fit value for $g(J / \psi)$ with the concept of $J / \psi-\omega-\phi$ mixing. In this approach one would write the reduced amplitude as

$$
g^{\operatorname{mix}}(J / \psi) \simeq M_{J / \psi}^{2} \tilde{\theta}_{\mathrm{c}} \sin \tilde{\theta}_{y} g_{\omega \rho^{0} \pi^{0}}\left(s=M_{J / \psi}^{2}\right) .
$$

The $\omega \rho^{0} \pi^{0}$ vertex function at $s=0$ can be estimated from chiral anomaly predictions for the coupling constants $g_{P \gamma \gamma}$ and vector meson dominancel:

$$
g_{\omega \rho^{0} \pi^{0}}(s=0) \simeq \frac{6 M_{\rho} M_{\omega}}{4 \pi^{2} \sqrt{2} f_{\pi} f_{\rho} f_{\omega}} .
$$

Using our values for the $J / \psi-\omega$ mixing parameters (30) and a monopole form for the $s$ dependence of the vertex function, $\Lambda^{2} /\left(M_{J / \psi}^{2}-\Lambda^{2}\right)$, we find that the fit value of $g^{\operatorname{mix}}(J / \psi)$ (13) is reproduced for $\Lambda \simeq 1 \mathrm{GeV}$. With regard to all the uncertainties encountered in an estimate like this, a value of about $1 \mathrm{GeV}$ for $\Lambda$ appears reasonable and is compatible with the typical inverse radius of a light hadronic system. Thus, we conclude that the small probability of the light-quark Fock components of the $J / \psi$ as estimated by $J / \psi-\omega-\phi$ mixing, suffices to generate the large $J / \psi \rightarrow P V$ branching ratios seen in experiment since it is overcompensated by the very large soft $\omega, \phi \rightarrow P V$ transition.

\footnotetext{
${ }^{7}$ This approach has been used to predict the radiative $P V$ transition, see 44] and references therein. Kramer, Palmer and Pinsky investigated the $\omega \rho^{0} \pi^{0}$ vertex on the basis of effective Lagrangians. Their numerical results are in agreement with Eq. (15). They also found $\left|g_{\omega^{\prime} \rho^{0} \pi^{0}}(s=0)\right| \ll\left|g_{\omega \rho^{0} \pi^{0}}(s=0)\right|$ which is in line with our assumption $g^{\operatorname{mix}}\left(\Psi^{\prime}\right) \ll g^{\operatorname{mix}}(J / \psi)$.
} 


\begin{tabular}{|c|c|c|c|}
\hline$V V$ & $c_{V V}^{\operatorname{mix}}$ & $10^{3} \cdot \mathcal{B}_{\mathrm{th}}$ & $10^{3} \cdot \mathcal{B}_{\exp }$ \\
\hline$\rho \rho$ & $\overline{1}$ & 16 & $25 \pm 8$ 46] \\
\hline$\omega \omega$ & 1 & 5.2 & $\begin{array}{l}<3.1 \text { 44 } \\
<6.3\end{array}$ \\
\hline $\bar{K}^{*} \bar{K}^{*}$ & $\left(y+\sqrt{2} \cot \left(\phi_{P}-\theta_{8}\right)\right) / 2$ & 11.1 & $8.2 \pm 3.9$ \\
\hline$\phi \phi$ & $y \sqrt{2} \cot \left(\phi_{P}-\theta_{8}\right)$ & 1.4 & $\begin{array}{l}9.0 \pm 5.0 \\
7.1 \pm 2.2 \\
3.1 \pm 1.2\end{array}$ \\
\hline
\end{tabular}

Table 5: Flavor factors (quoted for single channels) of decay amplitudes as well as experimental and theoretical branching ratios for $\eta_{\mathrm{c}} \rightarrow V V$ summed over all charge states. The quoted theoretical values correspond to a fit with $g^{\operatorname{mix}}\left(\eta_{\mathrm{c}}\right)=0.26 \mathrm{GeV}$; the mixing parameters for the pseudoscalar mesons are $\phi_{P}=39.3^{\circ}, \theta_{8}=-21.2^{\circ}$ and $y=0.81$ [20].

\section{Decays $\eta_{\mathrm{c}} \rightarrow V V$}

We write the helicity amplitudes for the $\eta_{\mathrm{c}} \rightarrow V V$ decays in a similar fashion as in Eq. (4) and parametrize the invariant amplitude analogously to Eq. (10) as

$$
A_{V V}\left(\eta_{\mathrm{c}}\right)=c_{V V}^{\operatorname{mix}} g^{\operatorname{mix}}\left(\eta_{\mathrm{c}}\right),
$$

with the decay width given by

$$
\Gamma\left(\eta_{c} \rightarrow V V\right)=\frac{1}{32 \pi} \frac{\varrho_{V V}\left(\eta_{c}\right)}{M_{\eta_{c}}}\left|A_{V V}\right|^{2}
$$

We assume that the $\eta_{\mathrm{c}}$ decay is mediated by the mixing of the $\eta_{\mathrm{c}}$ meson with the $\eta$ and $\eta^{\prime}$. The $\eta-\eta^{\prime}$ mixing parameters have already been quoted. The mixing mechanism leads to the flavor factors $c_{V V}^{\text {mix }}$ shown in Table $5\left(c_{\rho^{0} \rho^{0}}^{\text {mix }}=1\right)$. Electromagnetic contributions to $\eta_{\mathrm{c}}$ decays can only proceed via two photons and are thus negligible (see also footnote 4 ). Also a doubly OZI-rule violating decay mechanism should be strongly suppressed here since the production of light vector mesons through gluons is not enhanced by the $U(1)_{A}$ anomaly. The possibility of a decay through the c $\bar{c}$ Fock component of the light mesons is, as in the case of the $J / \psi$, ignored, see the discussion in Sec. 2.

A fit to the experimental branching ratios 5 [46, 47, 48] yields $g^{\operatorname{mix}}\left(\eta_{\mathrm{c}}\right)=0.26 \mathrm{GeV}$. The corresponding results are listed in Table 5. The strength of $g^{\operatorname{mix}}\left(\eta_{\mathrm{c}}\right)$ is compatible with the concept of mixing. To see this we first note that here the same vertex function, $g_{P V V}$, enters as in the $J / \psi \rightarrow P V$ decays in the vector meson mixing approach. Analogously to Eqs. (14,15) the reduced amplitude reads

$$
g^{\operatorname{mix}}\left(\eta_{\mathrm{c}}\right) \simeq M_{\eta_{\mathrm{c}}}^{2}\left|\theta_{\mathrm{c}}\right| \sin \left(\phi_{P}-\theta_{8}\right) g_{\eta_{q} \rho^{0} \rho^{0}}\left(s=M_{\eta_{\mathrm{c}}}^{2}\right)
$$

and the vertex function at $s=0$ is estimated by

$$
g_{\eta_{q} \rho^{0} \rho^{0}}(s=0) \simeq \frac{6 M_{\rho}^{2}}{4 \pi^{2} \sqrt{2} f_{q} f_{\rho}^{2}} .
$$

\footnotetext{
${ }^{8}$ Since the two results for the $\phi \phi$ channel quoted in Refs. 46, 48] are not compatible with each other, we have doubled the respective errors in the fit.
} 
Inserting the value $-1.0^{\circ}$ for the mixing angle $\theta_{c}$ that controls the light quark admixture to the $\eta_{c}$ as well as $f_{q}=1.07 f_{\pi}$ 20] and assuming again a monopole behavior of the vertex function the value $g^{\operatorname{mix}}\left(\eta_{\mathrm{c}}\right)=0.26 \mathrm{GeV}$ is reproduced for $\Lambda \simeq 1.3 \mathrm{GeV}$. This indicates a milder $s$ dependence of the vertex function in Eq. (18) than in Eq. (14). We stress that the mixing approach explains the order of magnitude of the $\eta_{\mathrm{c}} \rightarrow V V$ decay widths correctly. This is, to our opinion, a highly non-trivial fact. In tendency our results agree better with the experimental results quoted by the DM-2 collaboration 446] than with the ones found by MARK-III 47, 48]. Better data for the $\eta_{\mathrm{c}} \rightarrow V V$ channels are obviously needed. In analogy to the $\psi^{\prime} \rightarrow P V$ decays the $\eta_{\mathrm{c}}^{\prime} \rightarrow V V$ decays are expected to be strongly suppressed.

\section{Comments on decays into baryon-antibaryon pairs}

A perturbative calculation of the $J / \psi$ and $\Psi^{\prime}$ decays into baryon-antibaryon pairs has been carried through in Ref. [42] using the light-cone wave function for baryons proposed in Ref. [49]. In contrast to the $\chi_{\mathrm{cJ}}$ decays here it suffices to consider the color-singlet contributions only. The color-octet contributions are suppressed by powers of $1 / m_{\mathrm{c}}$ and start at the order of the charm-quark velocity, $v$, squared. Moreover, there is no obvious enhancement of the corresponding hard scattering amplitudes which appear with at least the same power of $\alpha_{s}$ as the valence Fock state contributions. Thus, despite the fact that $m_{\mathrm{c}}$ is not very large and $v$ not small $\left(v^{2} \simeq 0.3\right)$, it seems reasonable to expect small higher Fock state contributions to both these decays. One may, however, wonder what the strength of the mixing mechanism is. Since this mechanism is responsible for the large $P V$ branching ratios (see Sec. 2) it may also contribute to the baryon-antibaryon channels substantially and spoil the results presented in Ref. [42]. An estimate of the mixing contribution in analogy to Eqs. (14.15), using, for

instance, the $\omega$-nucleon coupling constants $g_{V \omega p \bar{p}}^{2} / 4 \pi=8.1 \pm 1.5$ and $g_{T \omega p \bar{p}}^{2} / 4 \pi=0.14 \pm 0.2$ [50], reveals that it amounts to less than $20 \%$ of the experimental width if the $s$ dependence of the $\omega p \bar{p}$ vertex function is at least as strong as that of the $\omega \rho \pi$ one. Since the $s$ dependence of a baryon vertex is likely to be stronger than that of a meson vertex the mixing contribution to the baryon-antibaryon channels is probably very small. Therefore the $J / \psi$ and $\Psi^{\prime}$ decays into baryon-antibaryon pairs seem to be under control of perturbative QCD. Indeed, the order $v^{0}$ perturbative results obtained in Ref. [42] agree well with experiment [8, 21]. As we emphasized in Sec. 2 the dominance of the perturbative contribution is further supported by the fact that the $\Psi^{\prime}-J / \psi$ ratio $\kappa_{B \bar{B}}$, defined in Eq. (2), is in agreement with unity (see Table 2) as is indicative for short-distance $c \bar{c}$ annihilation.

The hadronic helicity non-conserving decay $\eta_{\mathrm{c}} \rightarrow p \bar{p}$ may be estimated through $\eta_{\mathrm{c}}-\eta-\eta^{\prime}$

mixing. Using the coupling constant $g_{\eta N \bar{N}}^{2} / 4 \pi \simeq 1$ and $g_{\eta^{\prime} N \bar{N}} \simeq 0$ 45, 50 and equating the $s$ dependence of the $\eta N \bar{N}$ vertex with that of the $\eta V V$ one as determined from the fit discussed in Sec. 1 , we find $\mathcal{B}\left(\eta_{\mathrm{c}} \rightarrow p \bar{p}\right) \simeq 0.5 \cdot 10^{-3}$. Comparing with the experimental value of $(1.2 \pm 0.4) \cdot 10^{-3}$ [8] we are tempted to conclude that the mixing mechanism also controls the $\eta_{\mathrm{c}} \rightarrow p \bar{p}$ decays.

\section{Concluding remarks}

Exclusive charmonium decays constitute a laboratory for investigating power corrections and higher Fock state contributions as well as for studying the interplay of pQCD and soft mechanisms. That there are two distinct dynamical mechanisms at work can most easily be seen 
by comparison of $J / \psi \rightarrow P V$ and $\Psi^{\prime} \rightarrow P V$ decays. Their scaling with the charmonium decay constants is indicative for a short-distance mechanism (as, for instance, c $\overline{\mathrm{c}}$ annihilation into virtual gluons or photons) while a clear deviation from that scaling (as is seen for the helicity non-conserving $P V$ channels) signals the prominent role of a more peripheral soft mechanism. Several of the charmonium decays into pairs of pseudoscalar and/or vector mesons are forbidden by spin and parity invariance. Others, for instance the decays of the $C$-even charmonia into $P V$ channels, are under control of the electromagnetic decay mechanism because either $G$-parity or isospin conservation is violated for the channels involving non-strange mesons. Therefore, only the following classes of decays are actually possible: the helicity non-conserving $J / \psi\left(\Psi^{\prime}\right) \rightarrow P V$ and $\eta_{\mathrm{c}} \rightarrow V V$ decays which are not dominated by leading-twist pQCD; and the $\chi_{\mathrm{cJ}} \rightarrow P P, V V(J=0,2)$ decays which are accessible to a perturbative treatment and the $J / \psi\left(\Psi^{\prime}\right) \rightarrow P P, V V$ decays. The latter decays are of electromagnetic nature, the amplitudes being related to the time-like $P P$ or $V V$ electromagnetic form factors at $s=M^{2}\left(n^{3} \mathrm{~S}_{1}\right)$. One should, however, be aware of corrections to the electromagnetic decay mechanism from the isospin-violating part of pQCD. In principle, allowed by strong interactions are also the helicity non-conserving $\chi_{\mathrm{c} 1} \rightarrow V V$ decays. We however expect very small branching ratios for these channels. The decays of the $C$-even charmonia into the $P V$ channels proceed through $c \overline{\mathrm{c}}$ annihilation into two photons and are therefore strongly suppressed. In fact these decays as well as the $\chi_{\mathrm{c} 1} \rightarrow V V$ have not been observed in experiment as yet [8].

The perturbative analysis of the $\chi_{\mathrm{cJ}} \rightarrow P P$ decays has been carried through in Refs. [5, 23] and the results have been found to agree fairly well with experiment although, with regard to the recent BES data [33], some readjustment of the wave function parameters seems to be advisable. The $V V$ channels, on the other hand, have not been analyzed as yet. Note that the required light-cone wave functions of the vector mesons are not known to a sufficient degree of accuracy.

In the present paper we focus on the $J / \psi$ and $\Psi^{\prime}$ decays into the $P V$ channels as well as the $\eta_{\mathrm{c}} \rightarrow V V$ decays. We assume that in these cases the charmonium state decays dominantly by a soft mechanism through Fock components built up from light quarks only. This mechanism probes all quark-antiquark separations in the charmonium wave functions and therefore feels the difference between a $1 \mathrm{~S}$ and $2 \mathrm{~S}$-wave function. We model this mechanism by $J / \psi-\omega-\phi$ and $\eta_{\mathrm{c}}-\eta-\eta^{\prime}$ mixing, respectively, and argue that this contribution is negligible for $\Psi^{\prime}$ decays. The light vector and pseudoscalar mesons couple through vertex functions to the final state mesons. With a few parameters adjusted to experiment we find fair agreement with the many channels measured for $J / \psi\left(\Psi^{\prime}\right) \rightarrow P V$ and $\eta_{\mathrm{c}} \rightarrow V V$ decays. Most of the parameters have a simple, direct physical interpretation and their magnitudes can be estimated from physical considerations. Data of improved quality, in particular for the $\Psi^{\prime}$ and $\eta_{\mathrm{c}}$ decays are required for a more stringent test of our approach. A richer database for the vector-tensor channels in $J / \psi$ and $\Psi^{\prime}$ decays would also allow the extension of our mixing approach to this class of reactions.

\section{Acknowledgments}

We thank Thomas Teubner for a critical reading of the manuscript. 


\section{Appendix: Vector meson mixing}

In order to estimate the strength of the light-quark Fock components in the $J / \psi$ we consider $J / \psi-\omega-\phi$ mixing in parallel to the mixing of the pseudoscalar mesons. As in Refs. [20, 37] (see also [45) we start from the quark-flavor basis formed by the three states $\omega_{\mathrm{q}}, \omega_{\mathrm{s}}$ and $\omega_{\mathrm{c}}$ which are assumed to have the parton decompositions

$$
\begin{aligned}
\left|\omega_{\mathrm{q}}\right\rangle & =\Psi_{\mathrm{q}}|\mathrm{u} \overline{\mathrm{u}}+\mathrm{d} \overline{\mathrm{d}}\rangle / \sqrt{2}+\cdots, \\
\left|\omega_{\mathrm{s}}\right\rangle & =\Psi_{\mathrm{s}}|\mathrm{s} \overline{\mathrm{s}}\rangle+\cdots, \\
\left|\omega_{\mathrm{c}}\right\rangle & =\Psi_{\mathrm{c}}|\mathrm{c} \overline{\mathrm{c}}\rangle+\cdots
\end{aligned}
$$

where the ellipses stand for higher Fock states. The physical meson states are related to the basis (20) by an orthogonal transformation

$$
\left(\begin{array}{c}
\omega \\
\phi \\
J / \psi
\end{array}\right)=U\left(\phi_{V}, \tilde{\theta}_{y}, \tilde{\theta}_{\mathrm{c}}\right)\left(\begin{array}{c}
\omega_{\mathrm{q}} \\
\omega_{\mathrm{s}} \\
\omega_{\mathrm{c}}
\end{array}\right)
$$

where

$$
U=\left(\begin{array}{ccc}
1 & -\phi_{V} & -\tilde{\theta}_{c} \sin \tilde{\theta}_{y} \\
\phi_{V} & 1 & \tilde{\theta}_{\mathrm{c}} \cos \tilde{\theta}_{y} \\
\tilde{\theta}_{\mathrm{c}} \sin \tilde{\theta}_{y} & -\tilde{\theta}_{\mathrm{c}} \cos \tilde{\theta}_{y} & 1
\end{array}\right)
$$

$\left(U^{\dagger} U=1+\mathcal{O}\left(\tilde{\theta}_{\mathrm{c}}^{2}, \phi_{V}^{2}, \tilde{\theta}_{\mathrm{c}} \phi_{V}\right)\right)$. In Eq. (22) $\tilde{\theta}_{\mathrm{c}}$ and $\phi_{V}$ are considered as small quantities. $\tilde{\theta}_{\mathrm{c}}$ is small since the mixing between the light and the heavy sector is an effect of $\mathcal{O}\left(1 / m_{\mathrm{c}}^{2}\right)$ while the smallness of $\phi_{V}$ follows from the well-known fact that the $\omega$ and the $\phi$ are nearly ideally mixed [51].

The mass matrix in the quark-flavor basis is related to the (diagonal) mass matrix for the physical mesons by

$$
\mathcal{M}_{\mathrm{qsc}}^{2}=U^{\dagger}\left(\phi_{V}, \tilde{\theta}_{y}, \tilde{\theta}_{\mathrm{c}}\right) \operatorname{diag}\left[M_{\omega}^{2}, M_{\phi}^{2}, M_{J / \psi}^{2}\right] U\left(\phi_{V}, \tilde{\theta}_{y}, \tilde{\theta}_{\mathrm{c}}\right) .
$$

Following our previous work on the pseudoscalar mesons [20, 37], we parametrize the mass matrix as

$$
\mathcal{M}_{\mathrm{qsc}}^{2}=\left(\begin{array}{ccc}
m_{q q}^{2}+2 \tilde{a}^{2} & \tilde{y} \sqrt{2} \tilde{a}^{2} & \tilde{z} \sqrt{2} \tilde{a}^{2} \\
\tilde{y} \sqrt{2} \tilde{a}^{2} & m_{s s}^{2}+\tilde{y}^{2} \tilde{a}^{2} & \tilde{y} \tilde{z} \tilde{a}^{2} \\
\tilde{z} \sqrt{2} \tilde{a}^{2} & \tilde{y} \tilde{z} \tilde{a}^{2} & m_{c c}^{2}
\end{array}\right)
$$

where $\tilde{y}$ and $\tilde{z}$ allow for flavor symmetry breaking effects in the otherwise "democratic" mixing matrix whose strength, $\tilde{a}^{2}$, being controlled by quark-annihilation processes.

Comparing the expressions (23) and (24) we find the following six relations between the nine parameters

$$
\begin{aligned}
& m_{q q}^{2}+2 \tilde{a}^{2}=M_{\omega}^{2}, \\
& m_{s s}^{2}+\tilde{y}^{2} \tilde{a}^{2}=M_{\phi}^{2}, \\
& m_{c c}^{2}=M_{J / \psi}^{2}, \\
& \sqrt{2} \tilde{y} \tilde{a}^{2}=\left(M_{\phi}^{2}-m_{\omega}^{2}\right) \phi_{V},
\end{aligned}
$$




$$
\begin{aligned}
& \tan \tilde{\theta}_{y}=-\sqrt{2} / \tilde{y}, \\
& \tilde{\theta}_{\mathrm{c}}=-\sqrt{2+\tilde{y}^{2}} \tilde{z} \tilde{a}^{2} / M_{J / \psi}^{2} .
\end{aligned}
$$

One way to utilize these relations is to make use of flavor symmetry in order to fix the quark mass terms. Thus, one may use

$$
m_{q q}^{2} \simeq M_{\rho}^{2}, \quad m_{s s}^{2} \simeq 2 M_{K^{*}}^{2}-M_{\rho}^{2},
$$

in analogy to the pseudoscalar meson case [20]. This would allow the determination of the mixing parameters with the exception of $\tilde{z}$. However, we dismiss this method since it leads to values for the parameters which are instable under small corrections to the relations (26).

An alternative method that leads to robust values for the mixing parameters, is to take the mixing angle $\phi_{V}$ from experiment and to fix the parameters controlling flavor symmetry breaking in analogy to the pseudoscalar case by

$$
\tilde{y}=f_{\omega} / f_{\phi}, \quad \tilde{z}=f_{\omega} / f_{J / \psi}
$$

This is an assumption here in contrast to the pseudoscalar case where the divergences of the axial vector currents, embodying the $U(1)_{A}$ anomaly, relate the flavor symmetry breaking parameters to the meson decay constants. From the values of the decay constants quoted in Ref. [38], we obtain $\tilde{y}=0.80 \pm 0.04$ and $\tilde{z}=0.48 \pm 0.02$. The mixing ansatz (21) directly relates the ratio of the widths for the radiative $\phi-\pi^{0}$ and $\omega-\pi^{0}$ transitions to the mixing angle $\phi_{V}$ 19

$$
\frac{\Gamma\left(\phi \rightarrow \pi^{0} \gamma\right)}{\Gamma\left(\omega \rightarrow \pi^{0} \gamma\right)}=\phi_{V}^{2}\left[\frac{M_{\phi}}{M_{\omega}} \frac{\varrho_{\pi \gamma}(\phi)}{\varrho_{\pi \gamma}(\omega)}\right]^{3} .
$$

From the experimental decay widths [8] we find

$$
\phi_{V}=3.4^{\circ} \pm 0.2^{\circ}
$$

where the sign is chosen according to Eq. (25). This result is consistent with other determinations of that angle [51, 52].

Using Eqs. (27,29) and of course the masses of the physical vector mesons as input to Eq. (25), we obtain for the other mixing parameters

$$
\begin{aligned}
& \tilde{a}^{2}=0.022 \pm 0.002 \mathrm{GeV}^{2}, \\
& \tilde{\theta}_{y}=-59.8^{\circ} \pm 1.3^{\circ}, \\
& \tilde{\theta}_{\mathrm{c}}=-0.10^{\circ} \pm 0.01^{\circ} .
\end{aligned}
$$

The mixing strength, $\tilde{a}^{2}$ is much smaller than in the case of the pseudoscalar mesons [20] where it is dominated by the $U(1)_{A}$ anomaly. For completeness we note that an evaluation of the quark mass terms from these values for the mixing parameters leads to values which merely deviate by about $2 \%$ from those obtained via the flavor symmetry relations (26).

Our approach leads to the following quark content of the physical mesons

$$
\begin{aligned}
|\omega\rangle & =\left|\omega_{\mathrm{q}}\right\rangle-0.060\left|\omega_{\mathrm{s}}\right\rangle-1.5 \cdot 10^{-3}\left|\omega_{\mathrm{c}}\right\rangle, \\
|\phi\rangle & =\left|\omega_{\mathrm{s}}\right\rangle+0.060\left|\omega_{\mathrm{q}}\right\rangle-0.9 \cdot 10^{-3}\left|\omega_{\mathrm{c}}\right\rangle, \\
|J / \psi\rangle & =\left|\omega_{\mathrm{c}}\right\rangle+1.5 \cdot 10^{-3}\left|\omega_{\mathrm{q}}\right\rangle+0.9 \cdot 10^{-3}\left|\omega_{\mathrm{s}}\right\rangle .
\end{aligned}
$$




\section{References}

[1] A. Duncan and A. Mueller, Phys. Lett. B93, 119 (1980).

[2] S. J. Brodsky and G. P. Lepage, Phys. Rev. D24, 2848 (1981).

[3] V. L. Chernyak and A. R. Zhitnitsky, Nucl. Phys. B201, 492 (1982).

[4] T. Appelquist and H. D. Politzer, Phys. Rev. Lett. 34, 43 (1975).

[5] J. Bolz, P. Kroll and G. A. Schuler, Eur. Phys. J. C2, 705 (1998) hep-ph/9704378.

[6] G. T. Bodwin, E. Braaten and G. P. Lepage, Phys. Rev. D51, 1125 (1995) hepph/9407339].

[7] S. M. Wong, Eur. Phys. J. C14, 643 (2000) hep-ph/9903236; Nucl. Phys. A674, 185 (2000) hep-ph/9903221.

[8] Particle Data Group: Review of Particle Properties (C. Caso et al.), Eur. Phys. J. C3, 1 (1998).

[9] S. J. Brodsky and M. Karliner, Phys. Rev. Lett. 78, 4682 (1997) hep-ph/9704379.

[10] Y. Chen and E. Braaten, Phys. Rev. Lett. 80, 5060 (1998) hep-ph/9801226.

[11] L. J. Clavelli and G. W. Intemann, Phys. Rev. D28, 2767 (1983).

[12] S. S. Pinsky, Phys. Rev. D31, 1753 (1985) and Phys. Lett. B236, 479 (1990).

[13] X. Li, D. V. Bugg and B. Zou, Phys. Rev. D55, 1421 (1997).

[14] M. Suzuki, Phys. Rev. D57, 5717 (1998) hep-ph/9801284; Phys. Rev. D60, 051501 (1999) hep-ph/9901327.

[15] W. Hou and A. Soni, Phys. Rev. Lett. 50, 569 (1983); C. Chan and W. Hou, Nucl. Phys. A675, 367 (2000) hep-ph/9911423.

[16] S. F. Tuan, Commun. Theor. Phys. 33, 285 (2000) hep-ph/9903332; Y. F. Gu and S. F. Tuan, Nucl. Phys. A675, 404 (2000) hep-ph/9910423.

[17] P. Kroll, T. Pilsner, M. Schürmann and W. Schweiger, Phys. Lett. B316, 546 (1993) hep-ph/9305251.

[18] A. Seiden, H. F. Sadrozinski and H. E. Haber, Phys. Rev. D38, 824 (1988).

[19] A. Bramon, R. Escribano and M. D. Scadron, Phys. Lett. B403, 339 (1997) hep$\mathrm{ph} / 9703313$.

[20] T. Feldmann, P. Kroll and B. Stech, Phys. Rev. D58, 114006 (1998) hep-ph/9802409.

[21] F. A. Harris, hep-ex/9903036 and references therein.

[22] V. L. Chernyak and A. R. Zhitnitsky, Phys. Rept. 112, 173 (1984).

[23] J. Bolz, P. Kroll and G. A. Schuler, Phys. Lett. B392, 198 (1997) hep-ph/9610265. 
[24] J. Z. Bai et al. [BES Collaboration], Phys. Rev. Lett. 83, 1918 (1999) hep-ex/9901022.

[25] J. Z. Bai et al. [BES Collaboration], Phys. Lett. B424, 213 (1998); Erratum ibid. B438, 447 (1998).

[26] J. Z. Bai et al. [BES Collaboration], Phys. Rev. Lett. 81, 5080 (1998).

[27] V. Chernyak, hep-ph/9906387 [talk given at the International Workshop on $e^{+} e^{-}$collisions from $\phi$ to $J / \psi$, Novosibirsk (1999)] and references therein.

[28] V. A. Novikov, L. B. Okun, M. A. Shifman, A. I. Vainshtein, M. B. Voloshin and V. I. Zakharov, Phys. Rept. 41, 1 (1978).

[29] H. Meyer, Invited talk given at the 1979 Int. Symp. on Lepton and Photon Interactions at High Energies, Batavia, Ill., Aug 23-29, 1979 [DESY 79/81].

[30] B. Z. Kopeliovich, J. Nemchick, N. N. Nikolaev and B. G. Zakharov, Phys. Lett. B309, 179 (1993) hep-ph/9305225; P. Hoyer and S. Peigné, Phys. Rev. D61, 031501 hep$\mathrm{ph} / 9909519]$.

[31] G. Kramer, W. F. Palmer and S. S. Pinsky, Phys. Rev. D30, 89 (1984).

[32] J. Z. Bai et al. [BES Collaboration], Phys. Rev. D54, 1221 (1996).

[33] J. Z. Bai et al. [BES Collaboration], Phys. Rev. D60, 072001 (1999) hep-ex/9812016.

[34] R. Jakob, P. Kroll and M. Raulfs, J. Phys. G22, 45 (1996) hep-ph/9410304.

[35] P. Kroll and M. Raulfs, Phys. Lett. B387, 848 (1996) hep-ph/9605264.

[36] T. Feldmann and P. Kroll, Eur. Phys. J. C5, 327 (1998) hep-ph/9711231.

[37] T. Feldmann, P. Kroll and B. Stech, Phys. Lett. B449, 339 (1999) hep-ph/9812269.

[38] M. Neubert and B. Stech, in Heavy Flavours II, Eds. A.J. Buras and M. Lindner, World Scientific, Singapore hep-ph/9705292.

[39] A. Khodjamirian, Eur. Phys. J. C6, 477 (1999) hep-ph/9712451.

[40] T. Gousset and B. Pire, Phys. Rev. D51, 15 (1995) hep-ph/9403293; R. Jakob and P. Kroll, Phys. Lett. B315, 463 (1993) hep-ph/9306259 Erratum ibid. B319, 545 (1993).

[41] H. E. Haber and J. Perrier, Phys. Rev. D32, 2961 (1985).

[42] J. Bolz and P. Kroll, Eur. Phys. J. C2, 545 (1998) hep-ph/9703252].

[43] N. N. Achasov and V. V. Gubin, Phys. Rev. D61, 117504 (2000) hep-ph/9912393.

[44] A. V. Efremov and O. V. Teryaev, Sov. J. Nucl. Phys. 36, 140 (1982) [Yad. Fiz. 36, 242 (1982)].

[45] T. Feldmann, Int. J. Mod. Phys. A15, 159 (2000) hep-ph/9907491.

[46] D. Bisello et al. [DM2 collaboration], Nucl. Phys. B350, 1 (1991). 
[47] R. M. Baltrusaitis et al. [Mark-III Collaboration], Phys. Rev. D33, 629 (1986).

[48] Z. Bai et al. [MARK-III Collaboration], Phys. Rev. Lett. 65, 1309 (1990).

[49] J. Bolz and P. Kroll, Z. Phys. A356, 327 (1996) hep-ph/9603289.

[50] W. Grein and P. Kroll, Nucl. Phys. A338, 332 (1980).

[51] J.J. Sakurai, Phys. Rev. Lett. 9, 472 (1962); Phys. Rev. Lett. 19, 803 (1967).

[52] N. Isgur, Phys. Rev. D12, 3770 (1975); M. D. Scadron, Phys. Rev. D29, 2076 (1984). 